\title{
ARTICLE
}

\section{The design of a low activity laboratory housing a whole body counter consisting of large plastic scintillators and the work towards a flexible Monte Carlo calibration}

\author{
Jenny Nilsson* $^{*}$ and Mats Isaksson \\ The Sahlgrenska Academy, Institute of Clinical Sciences, Department of Radiation Physics, University of Gothenburg, Sweden
}

\begin{abstract}
A whole body counter consisting of four large plastic scintillator detectors (NE 102A, equivalent to BJ-400) has been used to measure spectra for a ${ }^{137} \mathrm{Cs}$ point source. The measured spectra were compared to Monte Carlo simulated spectra. The Monte Carlo code GATE v6.1 was used to simulate the energy deposition with the following optical transport in the scintillator material and detection of optical photons at the photocathode. MATLAB R2012a was used to calculate the multiplication in the photomultiplier tube. The results show the benefits of incorporating optical transport in the Monte Carlo simulations; the resolution in the simulated spectra changed drastically between energy deposition spectra and spectra of detected optical photons at the photocathode and; the measured spectra showed a source position dependence which was not noticed in the energy deposition spectra but first after the optical transport. A detailed description is given of the detector system and surrounding facilities because of their rather unique design.
\end{abstract}

Keywords: whole body counting; Monte Carlo calibration; optical transport; scintillation light; low activity laboratory; plastic scintillators; spectrometry; GATE

\section{Introduction}

After an accident or malicious act involving radioactive materials, where the general public has been contaminated or suspects contamination, is the capability to perform accurate and fast measurements on large groups of people of great importance. A suitable measurement method for contamination caused by ingestion of food products containing radioactive materials and/or from inhalation of radioactive particles or gases is whole body counting (WBC). At the University of Gothenburg two whole body counting spectrometry systems are housed in a low activity laboratory. System $\mathrm{I}$ is a $\mathrm{NaI}(\mathrm{Tl})$-detector system and System II consists of four large plastic scintillators. An on-going work is to develop Monte Carlo efficiency calibrations for both systems to be used in emergency situations. The purpose of a Monte Carlo calibration is to enable a quick startup of whole body measurements for various source distributions and energies.

The aims of this article are to: describe the construction of the low activity laboratory and show what System II is capable of; perform Monte Carlo simulations of energy deposition and optical transport in a plastic scintillator; calculate spectrum broadening after multiplication in a photomultiplier tube, PMT, and; present a comparison between measured and Monte Carlo simulated spectra for System II. Measurements

*Corresponding author. Email: jenny.nilsson@radfys.gu.se and simulations were done for a ${ }^{137} \mathrm{Cs}$ point source and the Monte Carlo code used was GATE v6.1 [1].

\section{Materials and methods}

\subsection{The WBC low activity laboratory}

The WBC facility was built in the late 60's and was first described by Sköldborn et al. [2]. The description of the facilities is still valid and drawings can be found in Sköldborn et al.'s work, but the detector electronics are continuously being replaced. The facilities are located in a bunker partly below ground. The roofs and walls are made of low activity iron concrete and the two separate WBC systems are housed inside a twin steel chamber, resting on an slab of Hoforsite concrete (Hoforsite is a low activity mineral from Hofors, Sweden). The chamber doors, roof and walls are made of $15.2 \mathrm{~cm}$ thick armor plates, resting on an additional $5.1 \mathrm{~cm}$ plate of the same metal (taken from the battleship 'Vanguard' and the cruiser 'Sheffield', both pre-1945, since metal from a later date contains small amounts of ${ }^{60} \mathrm{Co}$ ). The interior walls in both rooms are lined with $3 \mathrm{~mm}$ lead cladding, taken from old church roofs, to reduce backscattered photons from the human being measured.

The ventilation system serves only the low activity laboratory and the air is received trough a drum five stories high and HEPA-filtered to remove dust and other particles larger than $1 \mu \mathrm{m}$ in diameter. The air pressure is kept somewhat higher than in adjoining rooms to 
prevent gaseous radioactive elements, such as radon and radon daughters, from entering the laboratory. The humidity is constant and temperature variations are within the interval $\pm 0.5{ }^{\circ} \mathrm{C}$. Ventilation of the steel rooms is accomplished by a fan capable of eight complete air changes per hour, and the air pressure in the steel rooms slightly exceeds that in the remaining laboratory premises. The choice of building materials and ventilation thus ensures a low background with an ambient dose equivalent rate of $0.06 \mu \mathrm{Sv} / \mathrm{h}$ in the low activity facilities and $0.01 \mu \mathrm{Sv} / \mathrm{h}$ inside the steel rooms.

\subsection{Detector systems}

System II, used in this work, consists of four large plastic scintillators, NE 102A (the now obsolete product name $\mathrm{NE} 102 \mathrm{~A}$ is equivalent to $\mathrm{BC}-400$ [3]) each measuring $91.5 \times 76.0 \times 24.5 \mathrm{~cm}^{3}$, Figure 1 shows the schematics of System II. Each detector housing has a $0.397 \mathrm{~mm}$ copper window facing the patient bed. Two 12" $(30.5 \mathrm{~cm})$ PMTs (EM1 9545A) are mounted on each scintillator through a $16.5 \mathrm{~cm}$ long perspex light guide. For each detector the signals from the two PMTs are added and then amplified (Canberra Amplifier 816A). In the near future the amplifier and high voltage equipment will be replaced with up to date equipment. The signals from the upper and lower detectors are summed and recorded in a single spectrum using a pulse-height analyzer (Ortec ASPEC-927), but in this work the spectrum from one detector has been recorded to determine the individual detector response. The scintillator surfaces opposite the PMTs are wrapped in a reflecting aluminum foil. With two detectors above and two below a patient bed the measurement geometry is close to $3 \pi$. System II was initially designed for accurate and rapid measurements in clinical research, for example determination of total body potassium by measurements of ${ }^{40} \mathrm{~K}$ in the human body and nutritional research using different radionuclides as trace elements [4-8].

System I, with two $\mathrm{NaI}(\mathrm{Tl})$-detectors in a scanning bed geometry, offers a great versatility and has mostly been used for contamination controls of staff working with unsealed radiation sources as well as for metabolical studies [7, 9-11].

\subsection{Monte Carlo and MATLAB model of System II}

The Monte Carlo model of System II included the plastic scintillators, the reflecting aluminum foils, the copper windows, PMT photocathodes and parts of the detector housing and the patient bed. The light guides between the photocathodes and plastic scintillators were omitted and a photocathode volume measuring $0.0000025 \times 76.0 \times 24.5 \mathrm{~cm}^{3}$ was placed directly on the plastic scintillators short end. This simplification is further addressed in the discussion. The properties for the scintillation material were taken from the data sheet for BC-400 [12]. For each MeV deposited energy GATE samples the number of released optical photons from a Gaussian distribution with a mean value of 10000 optical photons. The spectrum of emitted light following energy deposition was defined for seven discrete wavelengths and their respective emission probabilities. To transport optical photons in GATE the user needs to define surface parameters. Based on test simulations (data not shown) the following settings were used. The surface between air and plastic scintillator was a dielectric-dielectric groundbackpainted $[13,14]$ surface with surface roughness parameter $\sigma_{\alpha}=0.1$ and $80 \%$ reflectivity, where all reflections were specular lobe reflections. The surface properties were independent of wavelength. The surface between aluminum foil, placed directly on the plastic scintillator, and the plastic scintillator was a dielectric-metal polished surface with $\sigma_{\alpha}=0.0$ and wavelength dependent reflectivity in the range 86.5 - $88.9 \%$ [15], where all reflections were specular lobe reflections. The optical photons were detected when reaching a surface placed on the photocathode. This surface was a dielectric-metal polished surface with $\sigma_{\alpha}=0.0$. The optical photons were detected with the wavelength dependent quantum efficiency for the PMT photocathode material S-11 [16]. GATE scores the number of photons per history detected at the surface but the wavelength information is not given in the output.

The spectrum broadening after multiplication in the PMT was calculated with MATLAB R2012a [17]. Each detected optical photon was assumed to produce one photoelectron. The gain $G$, the of number of electrons produced in a PMT per photoelectron, and its standard deviation $\sigma_{G} / G$ are given by Eq. (1) and Eq. (2)

$$
\begin{aligned}
& G=A \delta^{n} \\
& \sigma_{G}=G \frac{1}{\delta-1}
\end{aligned}
$$

where $A$ is the fraction of the photoelectrons collected by the multiplier structure, $\delta$ is the number of released electrons at each dynode per incoming electron and $n$ is the number of multiplication stages (dynodes) [3]. In this work $A=1, \delta=5$ [3] and $n=11$ [16] was used. A distribution of multiplicative events, such as the processes in a PMT, can be described by a lognormal distribution [18], where Eq. (1) and Eq. (2) give the expectation value $E \mid G]$ and standard deviation $s . d .[G]$. For each gamma history Eq. (3) and Eq. (4) was used to calculate the expectation value, $\mu$, and its standard deviation, $\sigma$, for a logarithmic distribution of $G$

$$
\begin{aligned}
& \mu=\ln (E[G])-\frac{1}{2} \ln \left(1+\frac{(s \cdot d[G])^{2}}{(E[G])^{2}}\right) \\
& \sigma=\sqrt{1+\frac{(s . d[G])^{2}}{(E[G])^{2}}}
\end{aligned}
$$

which was used as input in MATLAB's logninv function. The logninv returns the inverse of a cumulative lognormal function for a probability $p$. For each history a random probability between 0 and 1 was sampled and the returned inverse value was set as a new 
gain value, giving a final spectrum accounting for spectrum broadening caused by the multiplicative processes in a PMT.

\subsection{Measurement and simulation set-up}

A point source of ${ }^{137} \mathrm{Cs}(0.18 \mathrm{MBq}$, measurement time $30 \mathrm{~s}$ ) was placed on the patient bed at 3 positions along the middle of the patient bed: $30 \mathrm{~cm}$ (dot), $50 \mathrm{~cm}$ (diamond) and $70 \mathrm{~cm}$ (star) from the end of detector 1, see Figure 1. The same positions were used in the Monte Carlo simulations. The spectra were measured with detector 1 to investigate the individual detector response.

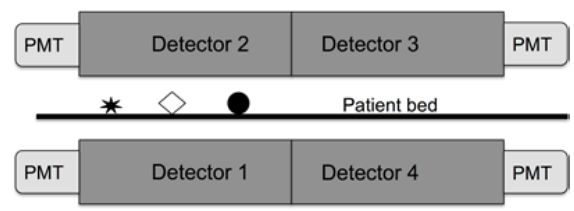

Figure 1. Schematics of System II. The markers dot, diamond and star show the source positions used for measurement and simulations. Each detector has 2 PMTs, in total 8 PMTs, but only 4 are seen since the PMTs are mounted next to each other on each detector.

\section{Results}

Figure 2 shows the spectra for the ${ }^{137} \mathrm{Cs}$ point source measured with detector 1 . The crosses are a 60 minutes background measurement, scaled to $30 \mathrm{~s}$ measurement time to give an indication of the background contribution. Figure 3 shows the Monte Carlo simulated energy deposition spectra and the spectra collected at the photocathode after optical transport in detector 1. Figure 4 shows the final simulated broadened spectra after multiplication in the PMTs.

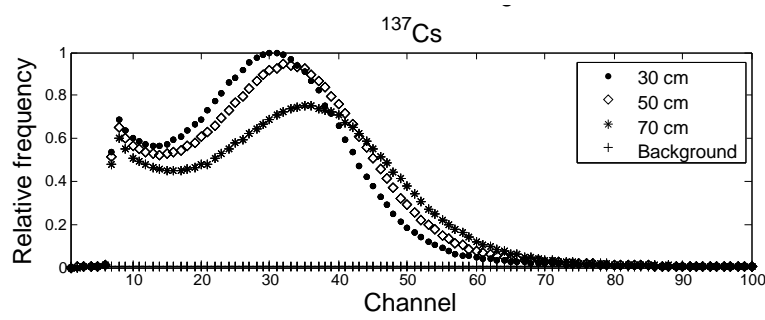

Figure 2. Measured spectra for a ${ }^{137} \mathrm{Cs}$ point source using detector 1. The source positions can be seen in Figure 1.

\section{Discussion and conclusions}

The results show that the generation of scintillation light and the following optical transport has a large impact on spectrum resolution. The energy deposition spectra in Figure 3 show little resemblance to the measured spectra seen in Figure 2. In the simulations where optical photons are collected by the photocathode the simulated spectra, Figure 3, begin to resemble the measured spectra in Figure 2. Figure 4 shows that the PMT does affect the spectrum resolution but the main reason for the characteristic shape of the plastic

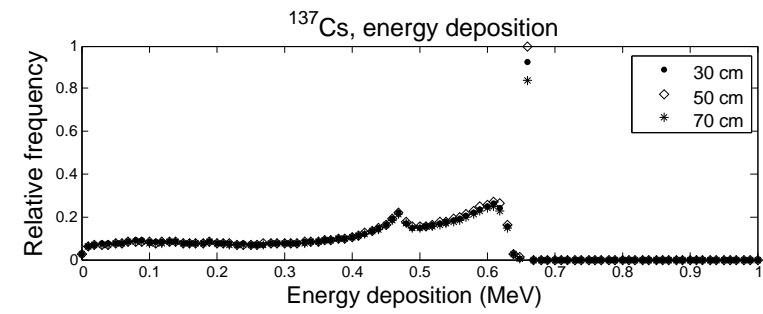

${ }^{137} \mathrm{Cs}$, optical transport following energy deposition

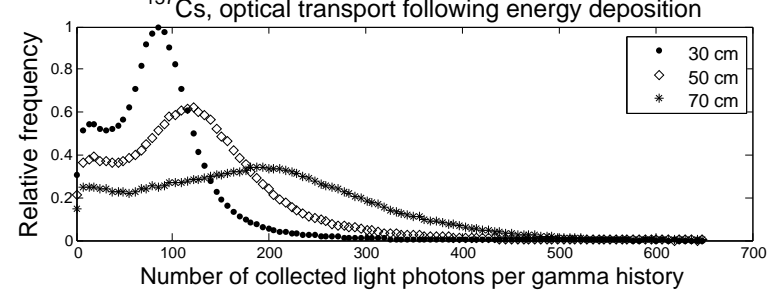

Figure 3. The upper spectra show the energy deposition in detector 1 for the ${ }^{137} \mathrm{Cs}$ point source and the lower show the optical photons detected by the photocathode. The source positions can be seen in Figure 1.

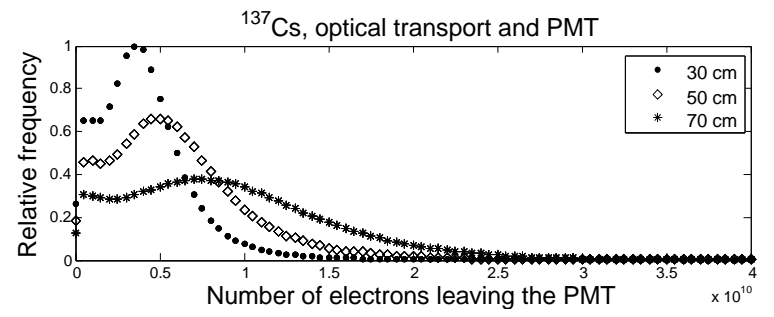

Figure 4. Simulated detector 1 spectra for a ${ }^{137}$ Cs point source after energy deposition followed by optical transport and multiplication in the PMTs. The source positions can be seen in Figure 1.

scintillator spectrum originates from the generation of scintillation light and optical transport. Unlike the energy deposition spectra, the optical spectra in Figure 3 show obvious source position dependence, which is also noted in the measured spectra. The position dependence for measured spectra has previously been reported, where the dependence was attributed to optical transport but it was not further investigated [19]. The main difference between the final simulated spectra in Figure 4, where optical transport and multiplication in the PMT were incorporated, and the measured spectra in Figure 2 is the magnitude of source position dependence. It seems like the simulations loses more optical photons the further away from the photocathode the source is placed and the simulated spectra therefore shows a larger position dependence compared to measured spectra. The loss of optical photons could also explain the difference in spectrum resolution seen in Figure 2 compared to Figure 4. In the simulations were the light guides omitted because their shape and properties were not fully known, but the optical transport in the light guides ought to be independent on source position. The model of the PMT is a simplification; not accounting for effects such as losses of photoelectrons between the photocathode and first dynode or electronic noise, but neither explains the differences between simulated and 
measured results with respect to source position. We believe that the losses of optical photons are due to an insufficient optical transport, either it may be the code itself or, more likely, the user defined material and surface properties. These properties are not always easily available or even measureable and in this work the surface and material properties has been found in the literature or determined after testing various parameters.

A major issue with optical transport is the simulation time; an energy deposition spectrum in Figure 3 took 3 minutes to simulate, whereas a scintillation light spectrum took 18 hours (using a MacBook Pro OSX 10.6.8 with a $2.53 \mathrm{GHz}$ Intel Core i5 processor). Still, by including optical transport in the Monte Carlo model we have gained a first insight into what causes the actual shape and resolution of a spectrum measured by a plastic scintillator.

When doing Monte Carlo simulations of spectrometer systems it is not uncommon to broaden the energy deposition spectrum to achieve a likeness to measured spectrum, e.g. [20, 21]. In general, a drawback with such an approach is that information of what causes the broadening is not fully known. For the specific case of large plastic scintillators would a broadening of the energy deposition spectra not account for the position dependence since it depends on optical transport. Therefore is such an approach not suitable in a Monte Carlo calibration for System II.

We believe that more work can be done in the optimization of the optical transport and the results show that adding optical transport and multiplication in the PMTs is the right way to go to achieve simulated spectra equivalent to measured spectra. A future work is therefore to look at the individual surface and material parameters to see how each affects the optical transport. When a good agreement is achieved for a point source more complex source configurations can be tested. With a knowledge and understanding of the entire signal chain and its effects on the final spectrum we can put more trust into a Monte Carlo calibration made for a source distribution that may not be as easily validated, as in the case of an emergency situation.

\section{Acknowledgements}

This project was financed by the Swedish Radiation Safety Authority and the Swedish Civil Contingencies Agency.

\section{References}

[1] S. Jan, D. Benoit, E. Becheva, T. Carlier, F. Cassol, P. Descourt, T. Frisson, L. Grevillot, L. Guigues, L. Maigne, C. Morel, Y. Perrot, N. Rehfeld, D. Sarrut, D.R. Schaart, S. Stute, U. Pietrzyk, D. Visvikis, N. Zahra and I. Buvat, GATE V6: a major enhancement of the GATE simulation platform enabling modelling of CT and radiotherapy, Phys. Med. Biol. 56 (2011), pp. 881-901.

[2] H. Skoldborn, B. Arvidsson and M. Andersson, A new whole body monitoring laboratory, Acta Radiol. Suppl. 13 (1972), pp. 233-41.

[3] G.F. Knoll, Radiation Detection and Measurement. $4^{\text {th }}$ ed., John Wiley \& Sons, Hoboken, N.J. (2010), pp.282-283, ISBN 9780470131480.

[4] M. Tengvall, L.Ellegard, N. Bosaeus, M. Isaksson, G. Johannsson and L. Bosaeus, Bioelectrical impedance spectroscopy in growth hormone-deficient adults, Physiol. Meas. 31 (2010), pp. 59-75.

[5] M. Alpsten, B. Arvidsson and L. Larsson, Measurements of K-40 in a whole body counter using coincidence technique for correction for Cs-134 contribution, 5th Nordic radioecology Seminar, Rättvik, Sweden, (1988).

[6] R.J. Brummer, L. Lonn, B.A. Bengtsson, H. Kvist, I. Bosaeus and L. Sjostrom, Comparison of different body composition models in acromegaly, Growth Regul. 6 (1996), pp. 191-200.

[7] M. Isaksson, I. Bosaeus, Y.Surac and M. Alpsten, Determination of potassium in the skeletal muscles by whole-body counting, Acta Diabetol. Suppl. 1 (2003), pp. 68-72.

[8] G. Ekenved, B. Arvidsson and L. Sölvell, Influence of food on the absorption from different types of iron tablets, Scand. J. Haematol. Suppl. 28 (1976), pp. 79-88.

[9] L. Davidsson, Å. Cederblad, B. Lönnerdal and B Sandström, Manganese retention in man: a method for estimating manganese absorption in man, Am. J. Clin. Nutr. 1 (1989), pp. 170-179.

[10]K. Fredlund, M. Isaksson, L. Rossander-Hulthén, S.Almgren and A.-S. Sandberg, Absorption of zinc and retention of calcium: dose-dependent inhibition by phytate, J. Trace. Elem. Med. Bio. 20 (2006), pp. 49-57.

[11]M. Isaksson, M. Alpsten, Å. Cederblad, L. Larsson and N. Pettersson, In vivo Identification and Localisation of Radioactive Contamination in the Human Body, Radat. Prot. Dosim. 89 (2000), pp. 317-319.

[12] Saint-Gobain Ceramics and Plastics Inc., BC-400, BC-404,BC-408, BC-412, BC-416 Premium Plastic Scintillators (2005).

[13]D.J. van der Laan, D.R. Schaart, M.C. Maas, F.J. Beekman, P. Bruyndonckx and C.W.E. van Eijk, Optical simulation of monolithic scintillator detectors using GATE/GEANT4. Phys. Med. Biol. 55 (2010), pp. 1659-1675.

[14]GATE- Users guide V6, available from: www.opengatecollaboration.org/, cited Feb 1, 2012.

[15] S. Riggi, P. La Rocca, E. Leonora, D. Lo Presti, G.S. Pappalardo, F. Riggi and G.V. Russo, GEANT4 simulation of plastic scintillator strips with embedded optical fibers for a prototype of tomographic system, Nucl. Intrum. Meth. A 624 (2010), pp. 583-590.

[16]EMI phptpmultiplier tubes, EMI Electronics LTD Valve Division, 30M/6-67 (PMT). 
[17]MathWorks Inc., MATLAB R2012a, (2012).

[18]H. Motulsky, Intuitive Biostatistics $2^{\text {nd }}$ ed., Oxford University Press, (2010), pp. 83-86, ISBN 9780199730063.

[19]J. Nilsson and M. Isaksson, A comparison between Monte Carlo-calculated and measured total efficiencies and energy resolution for large plastic scintillators used in whole-body counting, Radat. Prot. Dosim. 144 (2011), pp. 555-559

[20] E.R. Siciliano, J.H. Ely, R.T. Kouzes, J.E. Schweppe, D.M. Strachan and S.T. Yokuda,
Energy calibration of gamma spectra in plastic scintillators using Compton kinematics, Nucl. Intrum. Meth. A 594 (2008), pp. 232-243.

[21]G. Takoudis, S. Xanthos, A. Clouvas, M. Antonopoulos-Domis, C. Potiriadis and J. Silva, Spatial and spectral gamma-ray response of plastic scintillators used in portal radiation detectors; comparison of measurements and simulations, $\mathrm{Nucl}$. Intrum. Meth. A 599 (2009), pp. 74-81. 